\title{
Neue Behandlungsregelungen bei Bundeswehr und Bundespolizei
}

— Bei Polizeivollzugsbeamten der Bundespolizei gilt als Legitimation zur Behandlung im Notfall künftig der Dienstausweis. Ein Überweisungsschein kann innerhalb von vier Wochen nachgereicht werden. Besteht allerdings kein Notfall, muss weiterhin als Behandlungsvoraussetzung ein Überweisungsschein des Polizeiarztes vorgelegt werden, es sei denn, dass ausnahmsweise eine Krankenversicherungskarte ausgegeben wurde. Überweisungen vom Vertragsarzt an einen weiteren Vertragsarzt zur Untersuchung (Mit- oder Weiterbehandlung) sind weiterhin nicht möglich. Hier bleibt es dabei, dass der Patient grundsätzlich einen weiteren Überweisungsschein durch den Polizeiarzt einho- len muss. Die Ausnahmen werden aber ab 1. Januar 2013 erweitert und umfassen künftig auch Laborleistungen, zytologische Leistungen, Röntgenleistungen sowie anästhesiologische Leistungen im Rahmen ambulanter Operationen.

Auch bei der Versorgung von Soldaten der Bundeswehr gilt als Legitimation für eine Behandlung im Notfall ab 1. Januar 2013 die Vorlage des Truppen- beziehungsweise Dienstausweises. Auch hier kann der Überweisungsschein innerhalb von vier Wochen nachgereicht werden. Diese Regelung gilt auch dann, wenn ein Bundeswehrangehöriger außerhalb der Sprechzeiten vom Vertragsarzt behandelt wird. Die Regelungen bei Überweisungen vom
Vertragsarzt an einen weiteren Vertragsarzt zur Untersuchung sind identisch mit denen bei der Bundespolizei.

\section{MMW Kommentar}

Die Abrechnung dieser Leistungen erfolgt weiterhin über die zuständige Kassenärztliche Vereinigung. Ab 1. Januar 2013 gelten allerdings die regionalen Preise des EBM, also die regionale Eurogebührenordnung und nicht mehr wie bisher die alte ErsatzkassenGebührenordnung (E-GO). Dies bedeutet, dass die erbrachten Leistungen keiner Budgetierungs- oder Begrenzungsregelung unterworfen sind.

\section{Abrechnungsgenehmigungen gelten nicht rückwirkend!}

— Mit der Frage, ob bereits durchgeführte und abgerechnete Leistungen rückwirkend genehmigt werden können, hat sich das Sozialgericht Düsseldorf (SG) am 5. November 2012 befasst. Geklagt hatte ein Internist, der an der hausärztlichen Versorgung teilnimmt und die Fortsetzung seiner Genehmigung zur Abrechnung der "Zusatzpauschale Ösophago-Gastroduoendoskopie“ (Nr. 13400 EBM) erreichen wollte. Diese Leistung ist genehmigungspflichtig, da grundsätzlich dem fachärztlichen Versorgungsbereich zugeordnet. Das SG Düsseldorf hat nun entschieden, dass die Genehmigung nur für die Zukunft gilt. Es berief sich dabei auf die ständige Rechtsprechung des Bundessozialgerichts (BSG: u. a. Beschluss vom 3.2.2010, B 6 KA 20/09 B), wonach eine Rückwirkung nicht in Betracht kommt (Sozialgericht Düsseldorf, Urteil vom 5. November 2012, AZ: S 2 KA 56/12).

\section{MMW Kommentar}

Wichtig ist, dass nicht nur Erstgenehmigungen, sondern auch die Fortsetzung von Genehmigungen erst ab der Genehmigungserteilung wirksam werden. Es gilt deshalb, solche Verlängerungen rechtzeitig einzuholen.

Beachtenswert ist in diesem Zusammenhang auch ein weiteres Gerichtsurteil zur Abrechnung der Ösophago-Gastroduodenoskopie: Vertragsärzte haben demnach keinen Anspruch darauf, dass ihnen die Kosten für verwendete Einmal-Polypektomie-Schlingen erstattet werden. Das BSG hat am 15. August entschieden, dass ein Kostenerstattungsanspruch für ärztliche Instrumente nur dann in Betracht kommt, wenn die Kosten hierfür nicht bereits in den berechnungsfähigen Leistungen enthalten sind. Da die Nr. 13423 EBM aber die Kosten für mehrfach verwendbare Polypektomie-Schlingen enthält, scheidet nach Auffassung der Richter eine gesonderte Kostenerstattung für diese Instrumente aus. Unerheblich ist dabei, dass die Nr. 13423 EBM nur mehrfach verwendbare Polypektomie-Schlingen benennt und keine Einmal-Polypektomie-Schlingen. Laut BSG kann die Kostenregelung des EBM aber nicht dadurch umgegangen werden, dass der Vertragsarzt einmal statt mehrfach verwendbare Instrumente anwendet. Der Vertragsarzt würde sonst in unberechtigter Weise privilegiert, weil er die volle Vergütung für die Leistung einschließlich der kalkulatorisch enthaltenen Kostenanteile erhielte und zusätzlich die tatsächlichen Kosten erstattet bekäme (Bundessozialgericht, Urteil vom 15. August 2012, AZ: B 6 KA 34/11 R). 